\title{
Computer-assisted optics teaching at the Moscow Institute of Physics and Technology
}

Natalia Soboleva, Stanislav Kozel, Gennady Lockshin, M. Entin, K. Galichsky, et al.

Natalia N. Soboleva, Stanislav M. Kozel, Gennady R. Lockshin, M. A. Entin, K. V. Galichsky, P. L. Lebedinsky, P. M. Zhdanovich, "Computer-assisted optics teaching at the Moscow Institute of Physics and Technology," Proc. SPIE 2525, 1995 International Conference on Education in Optics, (13 October 1995); doi: 10.1117/12.224046

Event: SPIE's 1995 International Symposium on Optical Science, Engineering, and Instrumentation, 1995, San Diego, CA, United States 
Computer assisted optics teaching at the Moscow Institute of Physics and Technology

N.N.Soboleva, S.M.Kozel, G.R.Lockshin, M.A. Entin, K.V. Galichsky, P.L. Lebedinsky, P.M. Zhdanovich

Moscow Institute of Physics and Technology

\begin{abstract}
Traditional methods used in optics teaching lack clarity and vividness when illustrating abstract notions such as polarization or interference. Here's where computer models may help, but they usually show only a single phenomenon or process and don't let the student see the entire picture. For this reason at Moscow Institute of Physics and Technology was developed the courseware "Wave Optics on the Computer" consisting of a number of related simulations. It is intended for students studying optics at the Universities. Recently we have developed different simulations in optics for secondary school level. They are included as part of large computer courseware "PHYSICS by PICTURES".

The courseware "Wave Optics on the Computer" consists of nine large simulation programs and the textbook. The programs are simulating basic phenomena of wave optics. Parameters of optical systems can be varied by the user. The textbook contains theoretical considerations on studied optical phenomena, recommendations concerning work with computer programs, and, especially for those wishing to deeper understand wave optics, original problems for individual solution.

At the Moscow Institute of Physics and Technology the course "Wave Optics on the Computer" is used for teaching optics in the course of general physics. The course provides both computer assisted teaching for lectures support and computer assisted learning for students during a seminars in the computer classroom.
\end{abstract}




\section{WAVE OPTICS}

\subsection{Interference}

This program is designed to illustrate the fundamental laws of light wave interference. Various double-beam optical interference systems are simulated, i.e. Young's experiment, Fresnel bimirror, Michelson interferometer, interference in plane-parallel plate, Lloyd's mirror, Michelson stellar interferometer. The possibility of varying parameters of interference systems and the incident light wavelength is provided. Using Young's experiment as an example, the interference of partially coherent waves is simulated. The influence of light coherency properties and finite size of the sources on the interference pattern is studied.

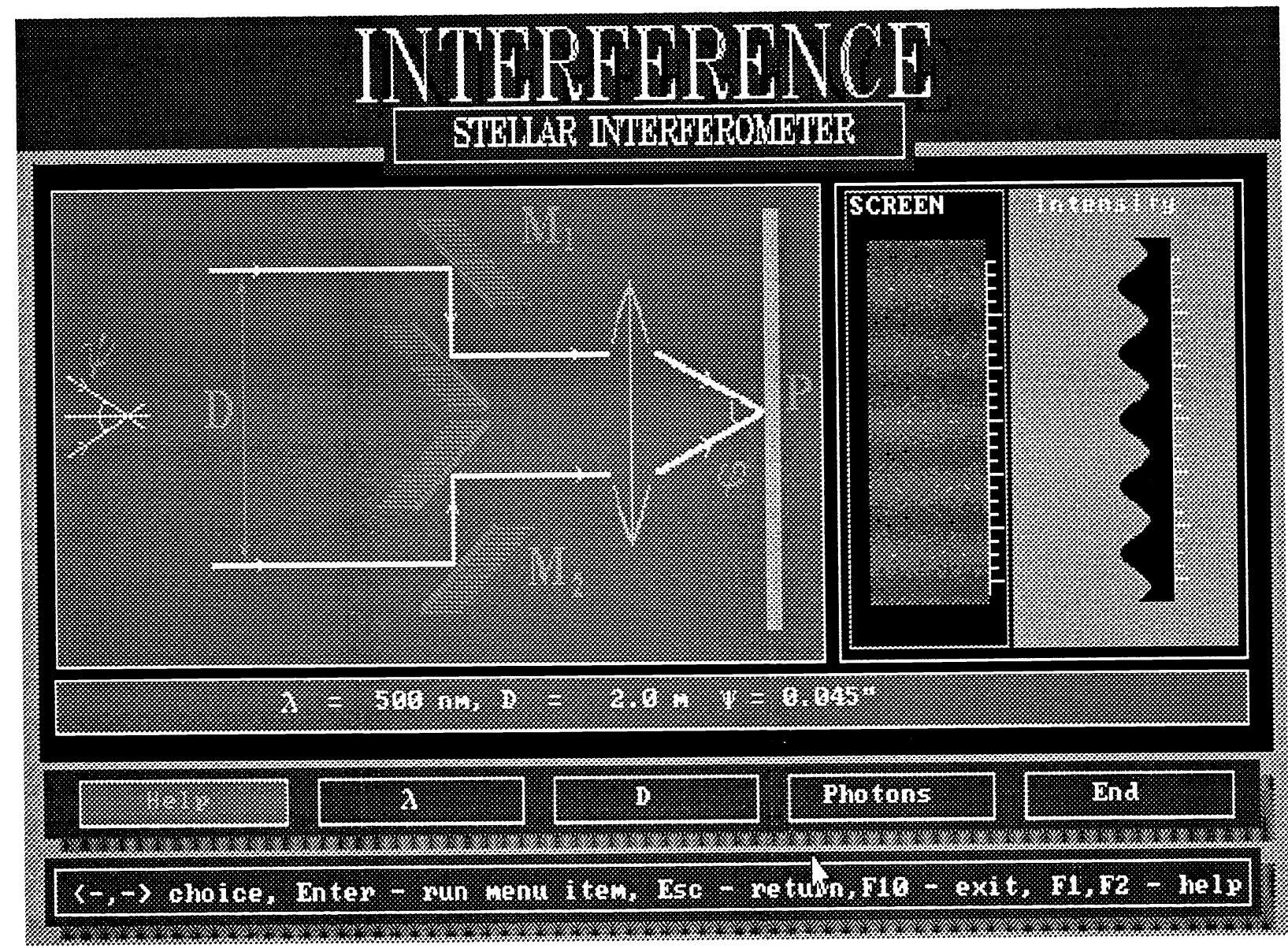




\subsection{Diffraction}

This program simulates diffraction of light waves on holes of different shape (round holes and slits). Both diffraction in the near zone (Fresnel diffraction) and far zone (Fraunhoffer diffraction) is covered by the program. Spatial distribution of light intensity in diffraction phenomena is simulated and displayed on the screen. Parameters of the model can be varied.

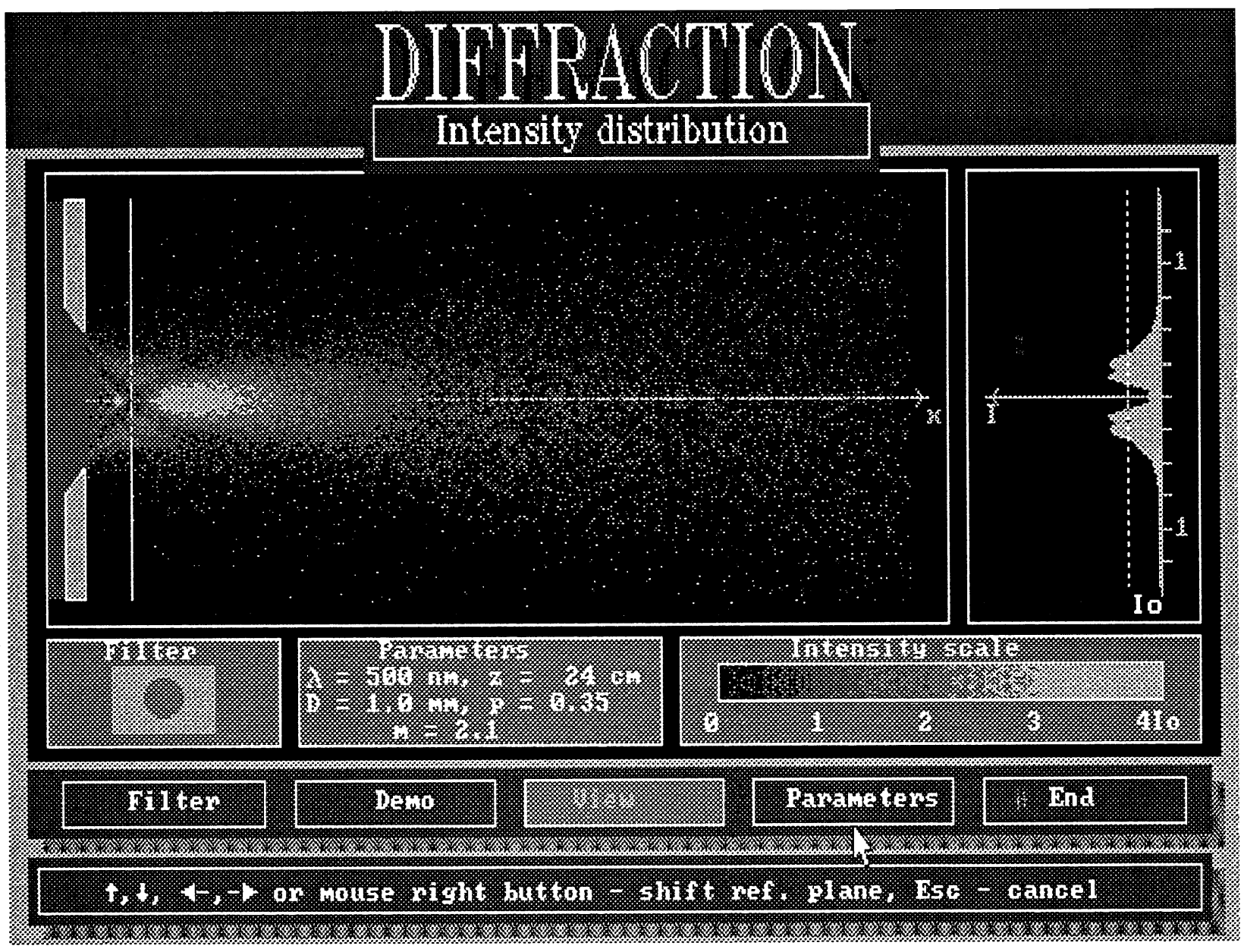




\subsection{Diffraction grating}

This program simulates the operating of diffraction grating. While various parameters of the model (such as wave length, period and number of slits, etc.) can be varied by the user the program calculates angular dispersion, dispersion range, resolution power etc, and presents the results in vivid pictures to be easily understood and remembered by students.

\subsection{Optical instruments}

This program illustrates chief principles of work of simple optical instruments. Main attention is paid to the influence of diffraction phenomena on the optical instrument operation. The work of human eye, lens, microscope and telescope is simulated. There is the possibility to change geometrical parameters of the devices and observe the results.

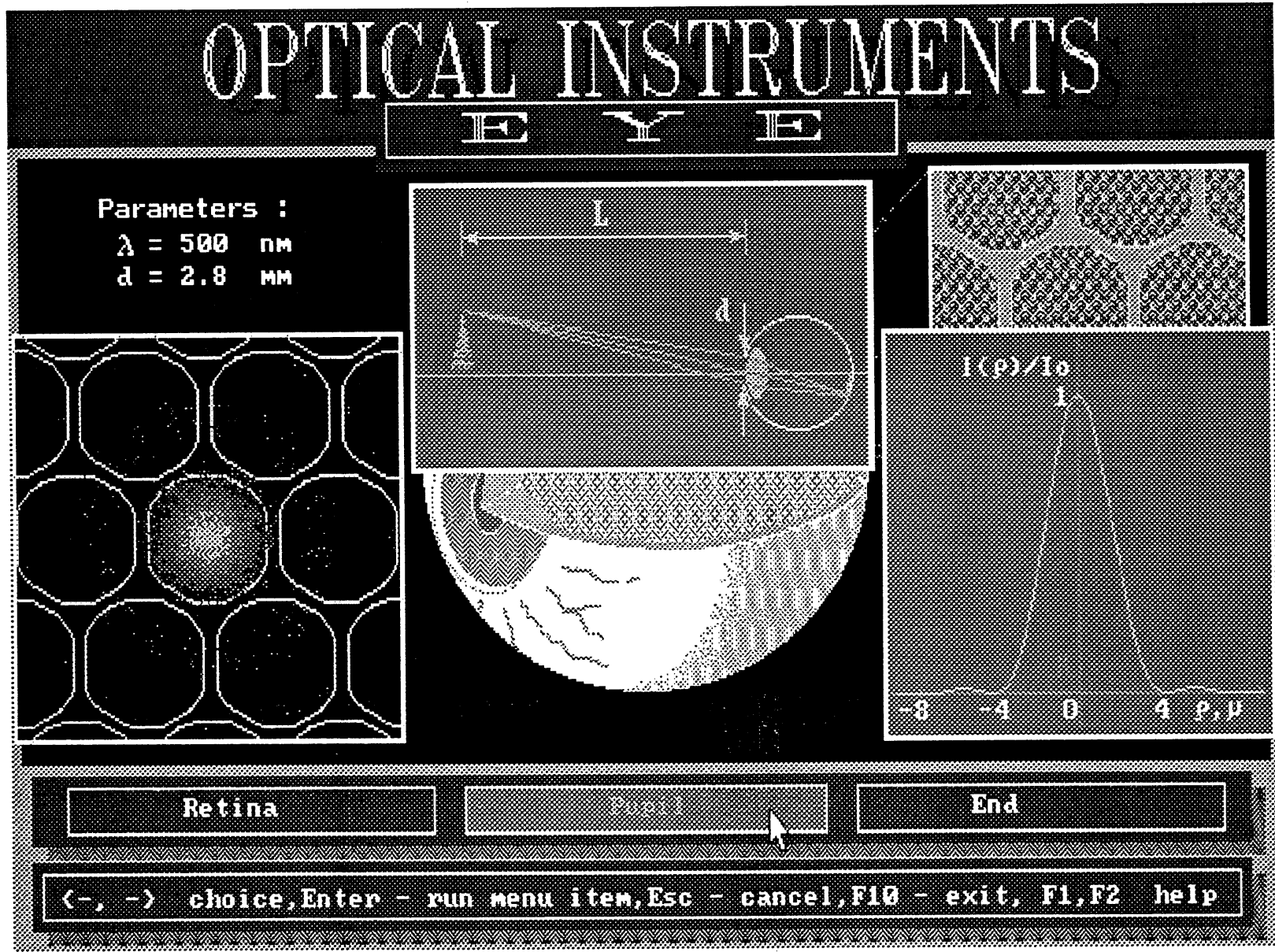




\subsection{Polarization}

This program demonstrates some properties of elliptically polarized light. Optical constructor contained in this program enables one to study operation of polarizing plates and double refracting plates. The program provides possibility to vary characteristics of input light and observe change of properties of output light beam.

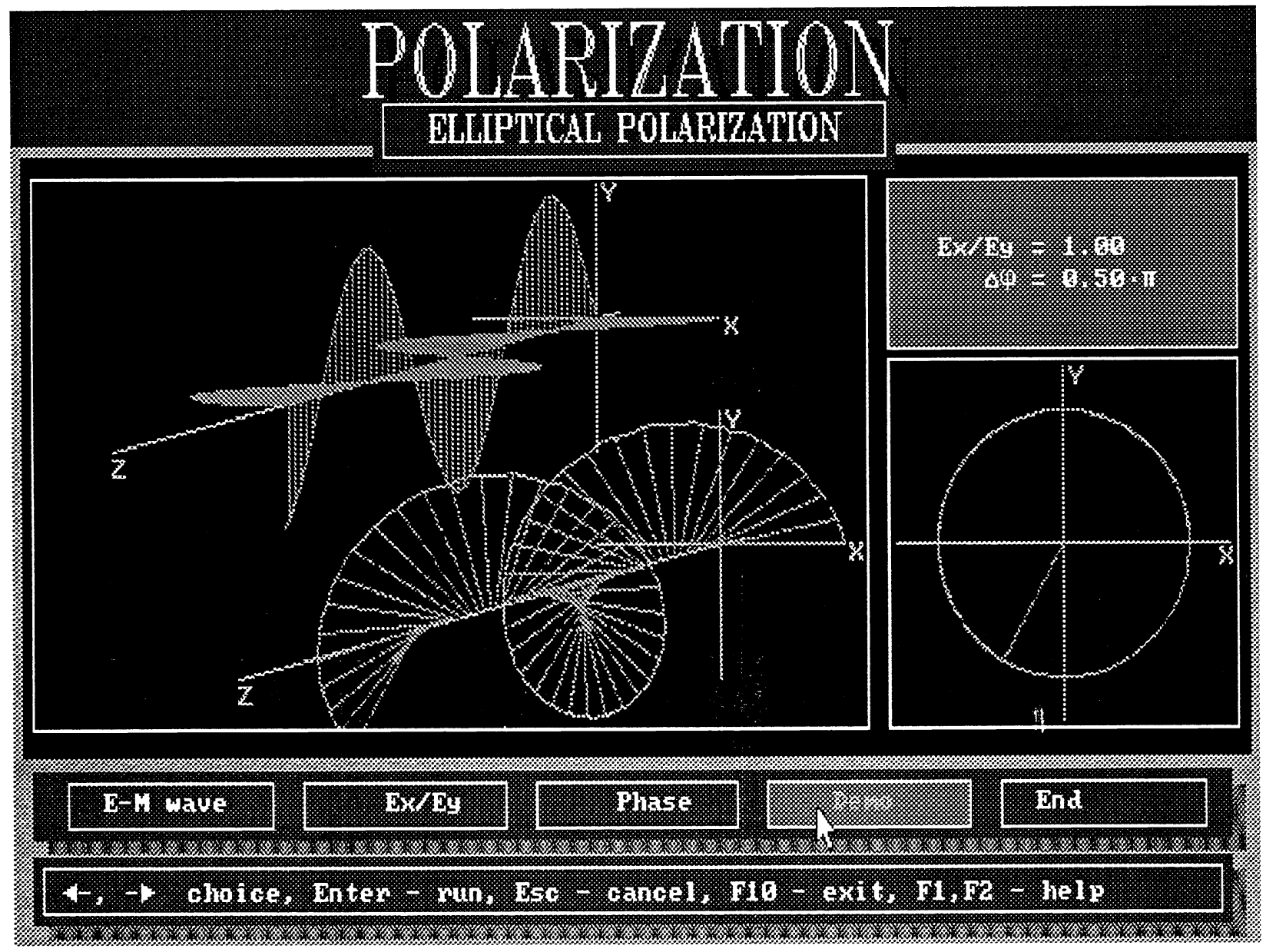

\subsection{Wave packets}

The program demonstrates phase and group velocities of light waves. Differences between phase and group velocities are explained on the example of superposition of three waves. Amplitudes and frequencies of these three waves can be varied in the program. Propagation of various wave packets is simulated in substances with different dispersion laws. Observation of normal and abnormal dispersions is provided. In some cases decay of wave packets can be observed. 


\section{ELEMENTS OF FOURIER OPTICS}

\subsection{Fourier analysis}

This program strives at explaining basic concepts of the theory of spectral expansions, such as direct and inverse Fourier transforms of some classes of periodical and aperiodic functions, discrete and continuous spectra, physical sense of positive and negative frequencies. The program allows for the parameters of studied functions to be varied. For periodic functions the program provides the possibility to observe single harmonic components and synthesize the former function with the help of inverse Fourier transform. For aperiodic functions the program allows to observe widening of spectra resulting from shortening of process duration and hence to clarify the uncertainty principle which is very important in physics of waves and vibrations. This program may be regarded as theoretical introduction to Fourier optics. It can be used as a separate program for education in vibrational phenomena, in electric circuits, mechanical systems etc.

\subsection{Fourier optics}

This program illustrates general ideas of Fourier optics on the bases of simulation of various optical problems, such as plane wave expansion of optical field, diffraction of light on various transparences, on amplitude and phase sinusoidal gratings, phenomenon of self-reproduction of light, principle of double diffraction, spatial filtration and multiplication of optical images.

\subsection{Two-dimensional Fourier transform}

This program provides the possibility to investigate spatial Fourier transform on the bases of Catron optical system. Fast Fourier Transform algorithm is used in calculations. The program allows for different optical transparencies to be chosen from the predefined set of pictures as well as new transparencies of arbitrary shape to be drawn by the user. After choice of the object the program carries out two-dimensional Fourier transform and builds up Fourier image as two-dimensional picture on display screen. Possibility of different manipulations with Fourier image is provided, such as masking of image with diaphragms and gratings, addition and subtraction of images etc. After editing Fourier image the program allows one to carry out second Fourier transform to get optical image and compare it with the original object. 


\section{D Fourier transform}

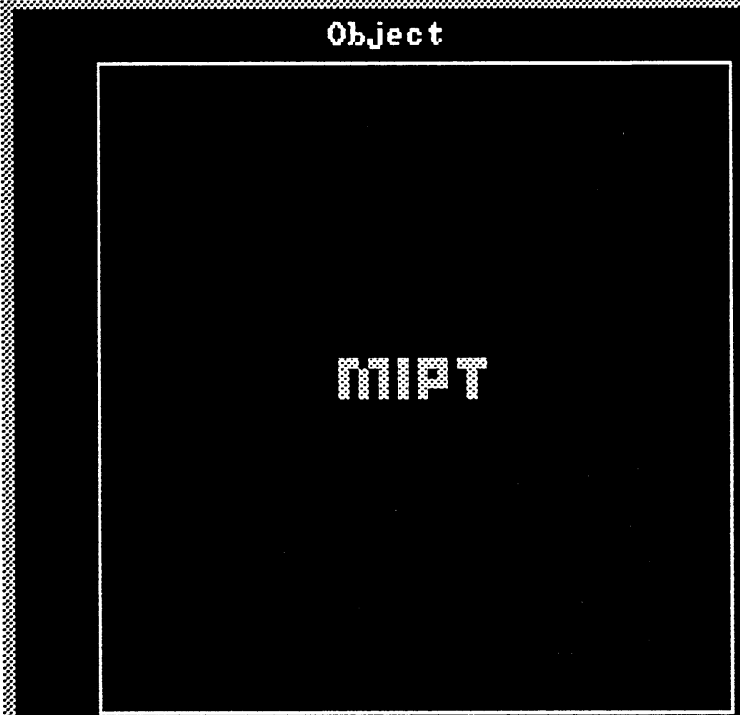

Level of intensity:1.000

\section{Fourien plane}

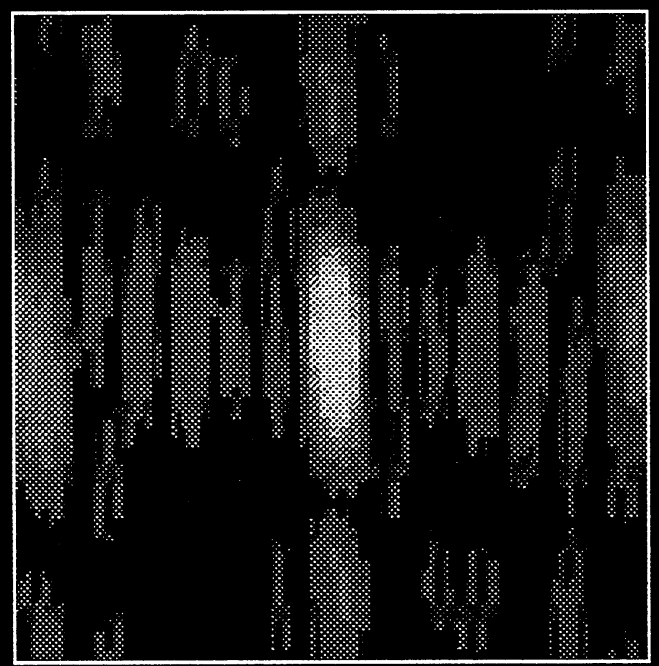

Level of intensity: 1028

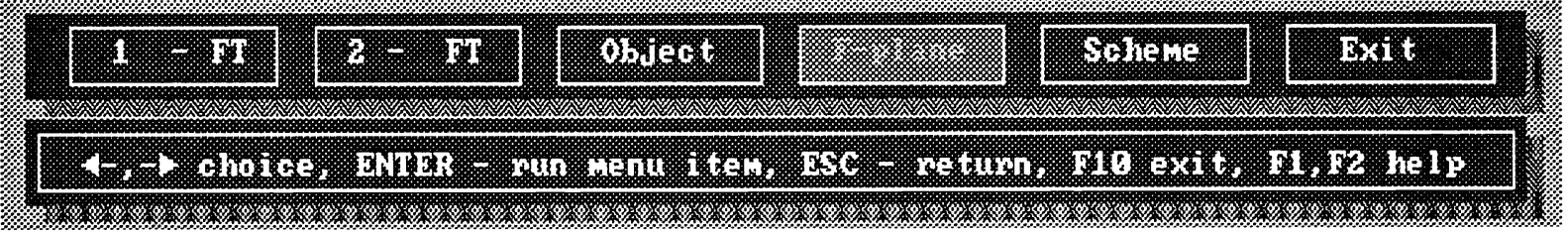

\section{PHYSICS BY PICTURES, PART "OPTICS”}

PHYSICS by PICTURES is educational computer courseware for both teachers and schoolchildren. It consists of reference book and a set of computer simulations from Mechanics, Thermodynamics and Molecular Physics, Electricity and Magnetizm, Optics, Quantum Physics as well as Historical Experiments examples. Some questions and different problems are included with possibility to enter answer and check it.

\subsection{Light and shadow}

There is visualized creation of shade and half-shade from the object and geometric light beams motion. It is possible to change size and position of the source, size and position of the obstacle. 


\subsection{Refraction and reflection of rays}

There is studied the law of light reflection from plane mirror. It is possible to change ray incidence angle and mirror angles. There are also studied laws of light refraction and reflection on the interface between air and glass in the computer experiment. It is possible to change ray incidence angle, glass refractive index, measure reflection and refraction angles. The phenomenon of total internal reflection on the interface between glass and air is studied.

\subsection{Thin lens}

The program demonstrates collecting and diverging lenses with different optical strength. It is possible to observe rays motion from remote source and look through the concept of focal point, focal length and optical strength of the thin lens. It is also possible to study the dependence between focal length of thin spherical lens and curvature of its surfaces and refractive indices of the lens and surrounding medium.

\subsection{Lens as an optical tool}

The simulation makes it possible to get images of objects in computer experiment with the aid of collecting and diverging lenses. It is also possible to choose the type of the lens and its focal length, to change object position and determine linear magnification.

\subsection{Optical constructor}

The program allows to construct image of object in optic system consisting of two lenses. It is possible to change object position, kind and parameters of lenses and the distance between them to determine linear magnification of two lens system. The program demonstrates rays motion in optical devices (microscope and telescope).

\subsection{Spherical mirror}

The program illustrates constructing the image of an object in concave and convex spherical mirrors. The concepts of focus and focal length of spherical mirror are studied. It is possible to change object position relative to the mirror, kind of the mirror and its curvature radius as well as determine linear magnification of image. 


\subsection{Eye as an optical tool}

There is studied motion of rays in an eye and position of image relative to retina depending on position of the object. It is possible to choose type of the eye - normal, near-sighted, far-sighted, look through the phenomenon of eye accomodation. Rays motion is presented for three different cases: eye is accomodated on the farthest accomodation point, eye is accomodated on the distant of normal vision, eye automatically changes its optical strength for the image of the object to remain on retina independently of position of the object. It is also possible to look through glasses operation.

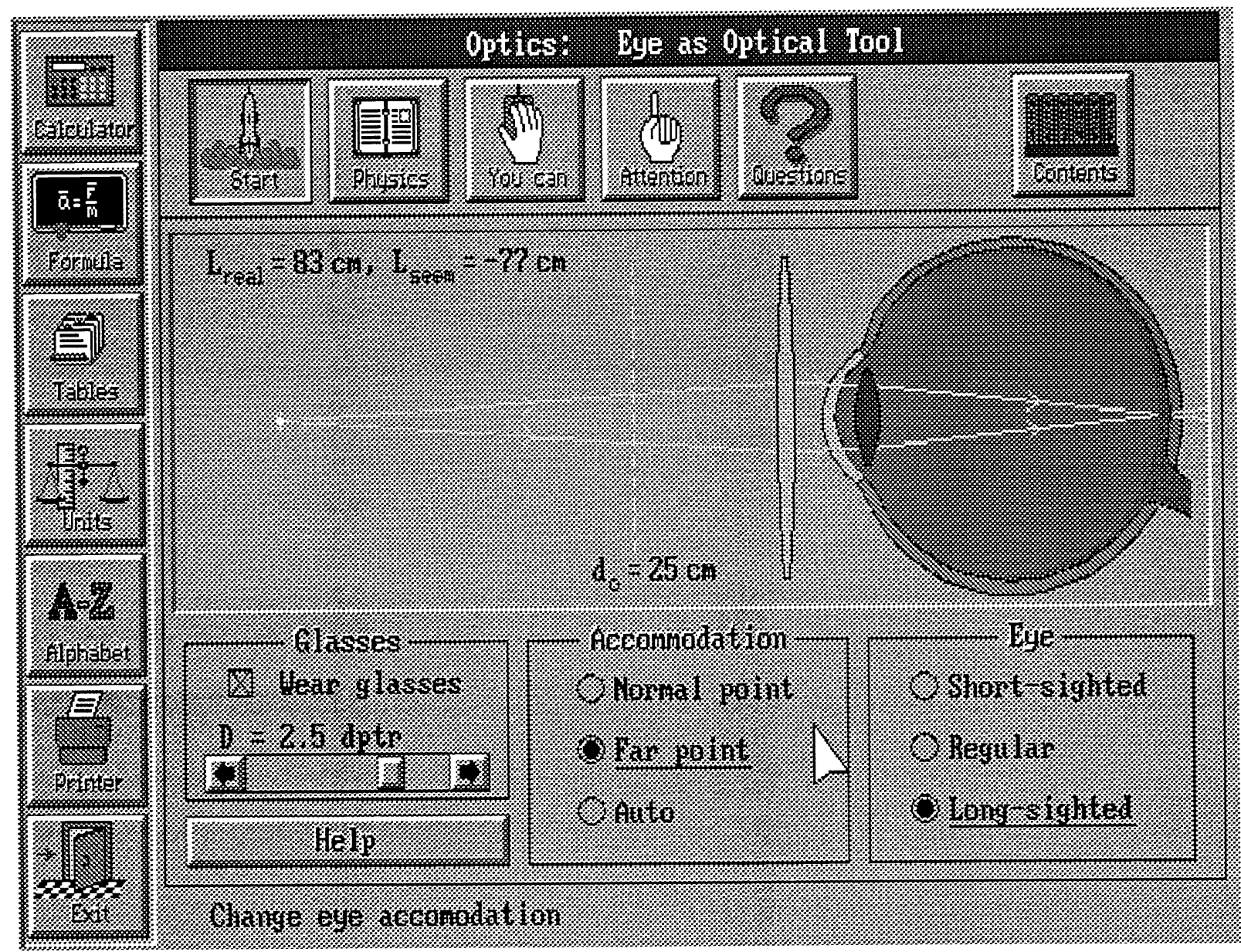




\subsection{Interference}

There is studied the phenomenon of interference on the example of Young's experiment. It is possible to observe interference fringes on the screen, plot of light intensity distribution and determine width of interference fridges. The simulation provides possibility of changing light wave length, distance between splits in Young's scheme and studing the influence of these parameters on interference pattern.

\subsection{Grating as an optical tool}

There is studied intensity distribution in diffraction pattern received from lattice with different number of splits. It is possible to observe rays motion, generate different orders of diffraction. The program allows to change number of splits in diffraction grating, its period and incident light wave length. Using the light consisting of two spectral lines it is possible to ensure that diffraction grating resolves them.

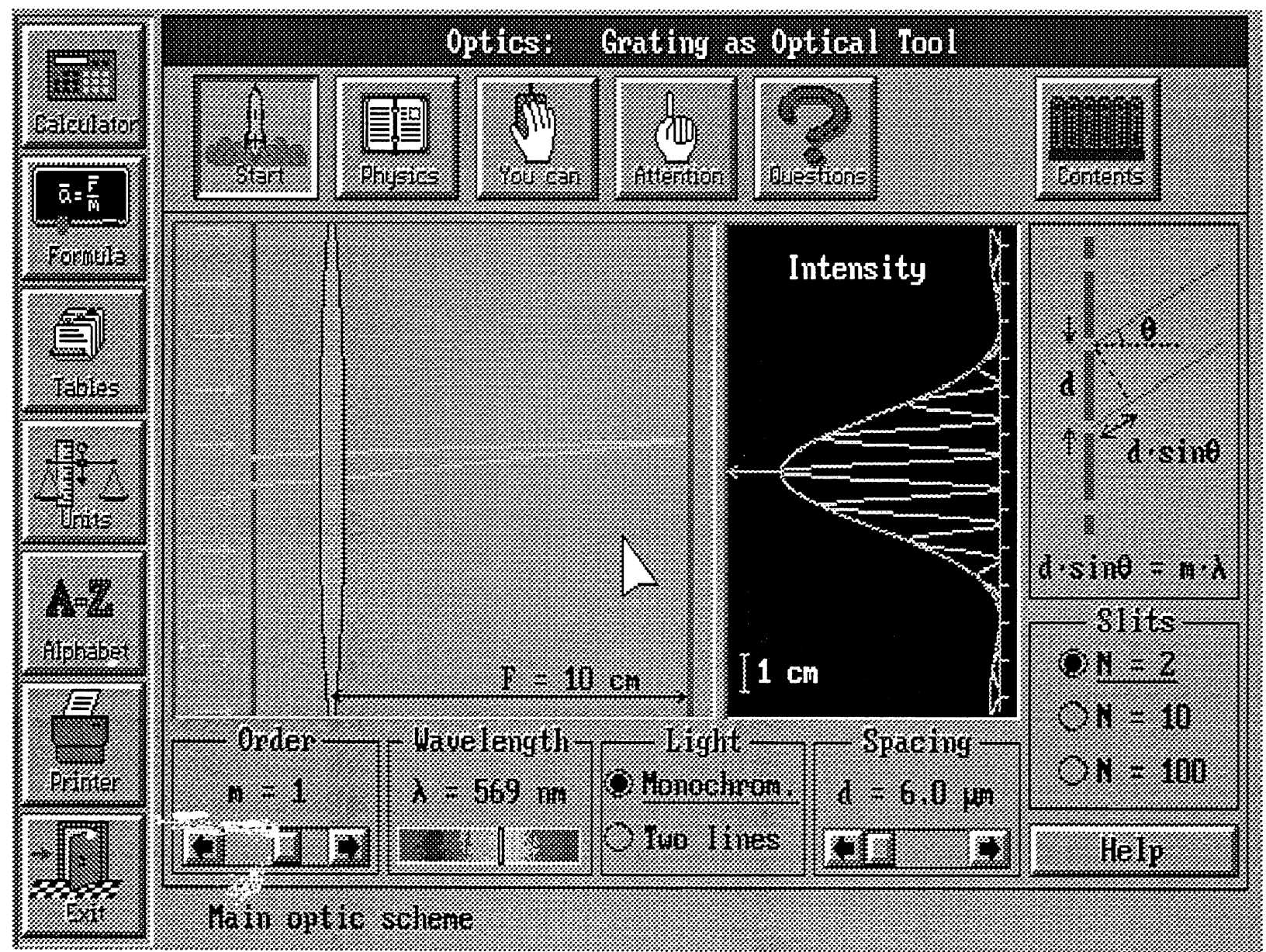




\subsection{Diffraction in lens focus}

There is observed image of the remote point source in the lens focus which is blurred because of light diffraction on lens aperture (on entrance hole). It is possible to change focal length, diameter of entrance aperture and light wave length.

\subsection{Fresnel zones}

The program allows to observe diffraction patterns from light diffraction on round apertures and round disks. It is possible to construct different zone plates, close or open any of first six ring Fresnel zones.

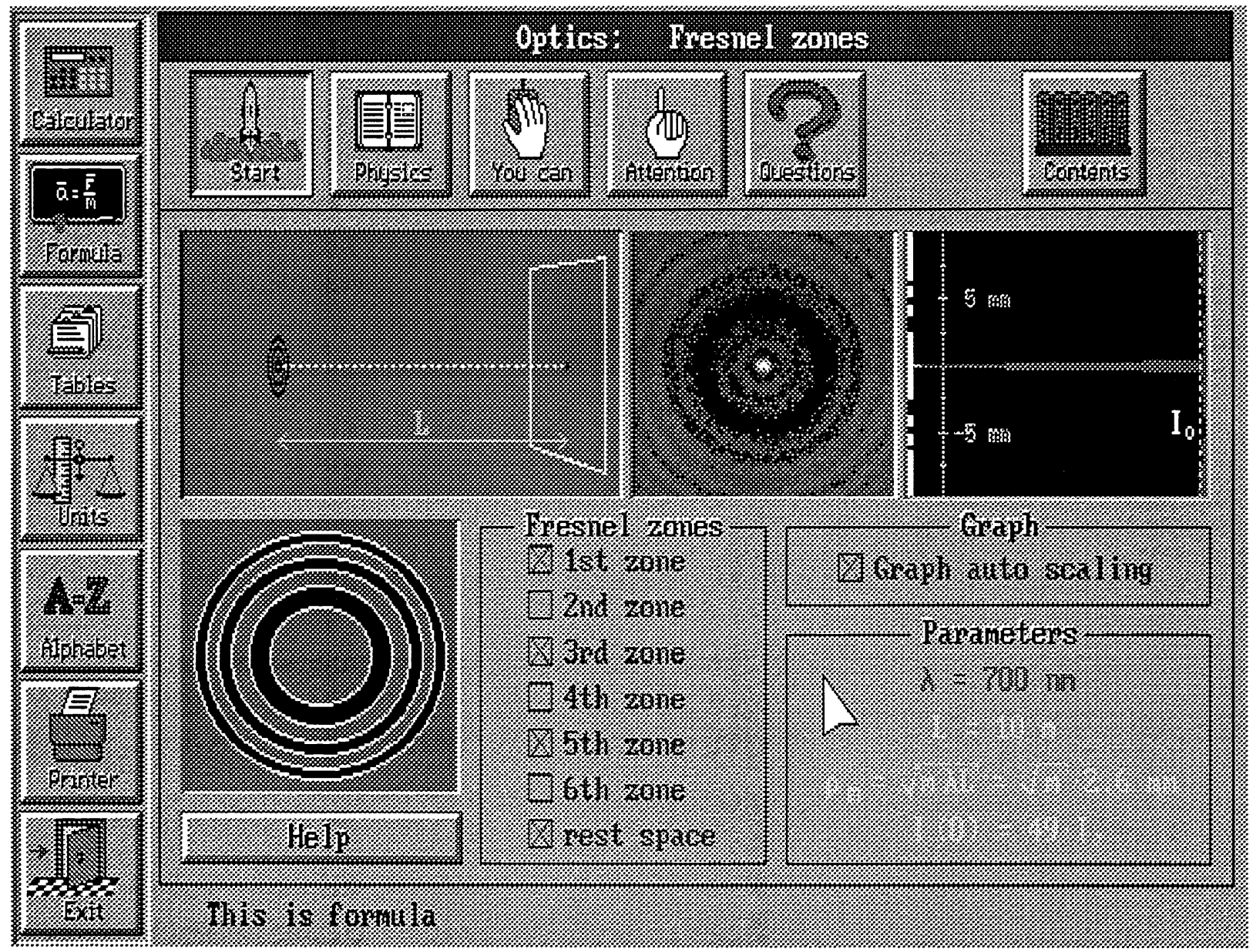




\subsection{Light polarization}

There is studied the phenomenon of light polarization with the aid of optical scheme consisting of light source, two polarizers and the screen. It is possible to change the orientation of permitted directions of polarizers, illustrate Malus law for the light consisting of different spectral components.

\subsection{Light speed. Michelson experiment}

The program demonstrates Michelson's experiment measuring the speed of light. It is possible to determine the speed of light changing angular velocity of octahedral mirror.

\subsection{Light dispersion}

The simulation allows to carry out computer experiment analogous to that of Newton's on resolution of white light in spectrum with the aid of a prism. It is possible to observe deviation of monochromatic light beams with different wave lengths by prism. The phenomenon of dispersion is also studied. 